\title{
Introgressive hybridization as a mechanism for species rescue
}

\author{
Marissa L. Baskett • Richard Gomulkiewicz
}

Received: 24 September 2010 / Accepted: 25 January 2011 / Published online: 22 February 2011

(C) The Author(s) 2011. This article is published with open access at Springerlink.com

\begin{abstract}
Rapid evolution on ecological time scales can play a key role in species responses to environmental change. One dynamic that has the potential to generate the diversity necessary for evolution rapid enough to allow response to sudden environmental shifts is introgressive hybridization. However, if distinct sub-species exist before an environmental shift, mechanisms that impede hybridization, such as assortative mating and hybrid inferiority, are likely to be present. Here we explore the theoretical potential for introgressive hybridization to play a role in response to environmental change. In particular, we incorporate assortative mating, hybrid inferiority, and demographic stochasticity into a two-locus, two-allele population genetic model of two interacting species where one locus identifies the species and the other determines how fitness depends on the changing environment. Simulation results indicate that moderately high values for the strength of assortative mating will allow enough hybridization events to outweigh demographic stochasticity but not
\end{abstract}

Article submitted to the Theoretical Ecology special issue in honor of Simon Levin.

Electronic supplementary material The online version of this article (doi:10.1007/s12080-011-0118-0) contains supplementary material, which is available to authorized users.

\section{L. Baskett $(\bowtie)$}

Department of Environmental Science and Policy,

University of California, Davis, One Shields Ave, Davis,

CA 95616-5270, USA

e-mail:mlbaskett@ucdavis.edu

R. Gomulkiewicz

School of Biological Sciences, Washington State University,

Pullman, WA 99164, USA so many that continued hybridization outweighs backcrossing and introgression. Successful introgressive hybridization also requires intermediate relative fitness at the allele negatively affected by environmental change such that hybrid survivorship outweighs demographic stochasticity but selection remains strong enough to affect the genetic dynamics. The potential for successful introgression instead of extinction with greater environmental change is larger with monogamous rather than promiscuous mating due to lower stochasticity in mating events. These results suggest species characteristics (e.g., intermediate assortative mating and mating systems with low variation in mating likelihood) which indicate a potential for rapid evolution in response to environmental change via introgressive hybridization.

Keywords Population genetic model - Introgression • Assortative mating - Demographic stochasticity •

Environmental change

"Yet neither discipline [population biology and ecosystem science] can afford to ignore the other: evolutionary changes take place within the context of ecosystems, and an evolutionary perspective is critical for understanding organisms' behavioral and physiological responses to environmental change."

-Levin (1992)

\section{Introduction}

Rapid evolution on ecological time scales can play a key role in species responses to environmental change, including human-driven changes (Frankham and 
Kingsolver 2004; Reznick and Ghalambor 2001; Stockwell et al. 2003). Theory indicates that the potential for adaptation to maintain population growth and therefore avert extinction depends on the rate of environmental change relative to the adaptive capacity as determined by genetic variation and selection strength (e.g., Lynch et al. 1991; Lynch and Lande 1993; Pease et al. 1989). The rate of environmental change is particularly acute in cases of sudden shifts, such as a sudden influx of a pollutant, habitat destruction, or extreme climatic events. Therefore, theory specific to adaptation in response to sudden environmental shifts suggests that constraints on the rate of genetic adaptation might limit its potential to play a role in response to such change, especially if population sizes are small, the change substantially affects fitness, or standing genetic variation is low (Gomulkiewicz and Holt 1995; Gomulkiewicz and Houle 2009; Boulding and Hay 2001; Orr and Unckless 2008).

One potential source of the genetic diversity necessary for adaptation to new or extreme environments is hybridization between sister species, which supplies genetic variation in the evolutionary and genomic context of a species' heritage (Rieseberg et al. 2003). Hybridization might lead to emergence of new hybrid species (as modeled by McCarthy et al. 1995; Buerkle et al. 2000; Duenez-Guzman et al. 2009), or hybridization might allow exchange of adaptive diversity between existing species. For the latter with both adaptation and the maintenance of separate species to occur, hybridization would have to take the form of introgression (where rare hybrids tend to backcross within populations, leading to limited gene transfer between distinct populations or species). Empirical examples suggest that introgressive hybridization can play a key role in adaptation to novel environments and environmental change in a diverse array of taxa (Anderson 1948; Arnold et al. 2008; Arnold and Martin 2010; Dowling and Secor 1997; Lewontin and Birch 1966). While introgressive hybridization subsequent to an environmental shift demonstrates the ability for related species to produce viable hybrid offspring, the presence of the separate species and lack of earlier introgression imply the existence of mechanisms that impede hybridization, such as hybrid inferiority and assortative mating. Accordingly, hybrid inferiority can explain why hybridization primarily takes place in the form of introgression (Anderson 1948).

In other words, dynamics that lead to rare and unsuccessful hybridization before an environmental shift might also allow for introgressive hybridization to occur after the environmental shift. Part of these dynamics will clearly depend on the change in relative fitness of different alleles for the introgressed gene during the environmental shift leading to an advantage to hybridization and hybrid backcrossing that partly outweighs overall hybrid inferiority (Dowling and Secor 1997). Furthermore, temporal variability is expected to increase the potential for hybridization and introgression because of changing selection (Dowling and Secor 1997). For example, introgressive hybridization and shifting hybrid fitness under environmental change is central to one of the best-documented cases of contemporary evolution: Grant and Grant's (2002) study of Darwin's finches. In this study, two finches, Geospiza fortis and Geospiza scandens, hybridize rarely due to assortative mating by bird song. An El Niño-driven change in seed composition that favored the beak morphology of hybrids caused an increase in hybrid fitness. Subsequent backcrossing of hybrids to the parental species led to the introgression of beak morphology from $G$. fortis to $G$. scandens, with bird song continuing to drive preferential mating within each species (Grant and Grant 2002, 2008).

In addition to the balance between hybrid inferiority and fitness changes, introgressive hybridization will depend on how these factors balance with assortative mating (Dowling and Secor 1997) and how that balance might shift with changing demographics. Specifically, consider a case where assortative mating reinforces distinct species before an environmental shift, and environmental change reduces the fitness of alleles present in only one of the species. Then that species will experience a large drop in numbers that the other species will not, and the disadvantaged species will have a higher likelihood of encountering the advantaged species. If mating depends on both probability of encounter and mating preference, the change in encounter rates during a period of low population size might lead to greater hybridization rates despite assortative mating (Wirtz 1999). In addition, the rare hybrids will be more likely to backcross than mate with other hybrids due to the demographics of encounter rates as well as assortative mating (as observed in Darwin's finches; Grant and Grant 2008). Once backcrossing with hybrids allows for introgression of the advantageous allele and an increase in population size, the assortative mating and any hybrid inferiority from other traits can again reinforce the separate species and prevent further hybridization. In support of this potential importance of demographically driven encounter rates to introgressive hybridization, gene flow between two species often occurs from the more common to the more rare species (Grant and Grant 2008).

While many population genetic models have provided insight into quantifying the role that assorta- 
tive mating can play in driving sympatric speciation and maintaining species-level diversity (e.g., Karlin and Scudo 1969; Felsenstein 1981; Barton 2001; Dieckmann and Doebeli 1999; for a thorough treatment of this topic see Coyne and Orr 2004; Gavrilets 2004), how assortative mating interacts with demographics to determine the potential for introgression remains less well understood. In addition, a deeper understanding of the roles of demographics and mating dynamics is necessary to determine under what conditions the general theoretical expectation of favorable alleles to spread in hybrid zones via introgression (Barton 2001) can occur rapidly enough to rescue a species that would otherwise go extinct following environmental change. In this study, we use a population genetic model that incorporates the interaction between assortative mating, demographics, and hybrid inferiority in the potential introgression of a gene between two species. In particular, this model investigates when (i.e., under what parameter values and assumptions reflective of different species characteristics) introgressive hybridization is likely to play a role in response to environmental change.

\section{Model description}

In addition to the potential importance of the demographics of mating described in the "Introduction," demographic stochasticity itself can readily outweigh genetic effects when determining the potential for extinction (Lande 1988). To understand the influence of assortative mating and demographic stochasticity on introgression and the potential for extinction in the absence of adaptive evolution, we incorporate both of these processes into the model. Then we present two comparisons of the capacity for introgressive hybridization that elucidate the roles of these processes: a stochastic version of the model to a deterministic version and a promiscuous mating system to a monogamous mating system which differs in the likelihood of mating success.

In order to explore the introgression of one gene or gene complex among the broader genome, the model uses a two-locus approach: The first locus serves as the species identifier and represents the broader genome, while the second locus represents the gene or gene complex at the focus of potential introgression. Each locus has two alleles, $\mathrm{A}$ and a for the first locus and $\mathrm{B}$ and $\mathrm{b}$ for the second locus; individuals are diploid for both loci. Because the first locus is the species identifier, AA individuals indicate members of species 1 , aa individuals indicate members of species 2 , and Aa individuals indicate hybrids. Therefore, introgression occurs in the model if a population initially comprised of entirely $\mathrm{AABB}$ and aabb individuals becomes a population comprised of $\mathrm{AABB}$ and aaBB individuals following a reduction in fitness for individuals carrying the $b$ allele.

To account for the role of demographics, the model follows the number of individuals with each genotype rather than the traditional population genetic approach of following proportions. Then the steps of the model in each (discrete) generation, described in more detail below, are: (1) mating determined by encounter probabilities, the strength of assortative mating, and the mating system; (2) inheritance, including possible recombination between the two loci; (3) density-dependent survivorship based on the strength of interaction between the two species; and (4) density-independent survivorship based on the fitness at both loci (Fig. 1). This last step models hard selection determined by the degree of hybrid inferiority and the relative fitness at the allele affected by environmental change in order to allow the potential for extinction when adaptation does not occur. In the version with demographic stochasticity, we implement the model as an individualbased simulation, where each individual mates, inherits a particular genotype, and survives with a given probability as defined below. In the deterministic version, these probabilities determine the proportion of the population that undergoes each process (mathematical details in ESM A).

\section{Mating}

In the stochastic model, mating occurs according to one of two mating functions, the "promiscuous mating function" and the "monogamous mating function." In both, the first step is the random selection of two individuals, an encounter event where the probability that a given genotype pair will encounter each other is the product of the proportion of each genotype in the population. Then whether a mating occurs given the encounter depends on the genotypes at the first locus and the assortative mating parameter $n$. Specifically, while individuals with the same genotype at the first locus $(\mathrm{AA} \times \mathrm{AA}$, aa $\times$ aa, or $\mathrm{Aa} \times \mathrm{Aa})$ always mate given an encounter, individuals of different species $(\mathrm{AA} \times \mathrm{aa})$ will mate with probability $n$. Hybrids are intermediates: The probability of a hybrid mating a homozygote of either type (Aa $\times$ aa or AA) given an encounter is $(1+$ $n) / 2$. In other words, the assortative mating parameter $0 \leq n \leq 1$ is the probability of an AA individual and an aa individual mating given an encounter, where $n=0$ for complete reproductive isolation, $n=1$ for random 
Fig. 1 Outline of the model dynamics. Parameters related to each step are in bold; see Table 1 for parameter definitions. $N_{i j}^{\prime}$ indicates the number of post-reproduction individuals with genotype $i j \in\{\mathrm{AA}, \mathrm{Aa}$, aa $\}$. Note that the symbols and colors correspond to those used in Figs. 2-5

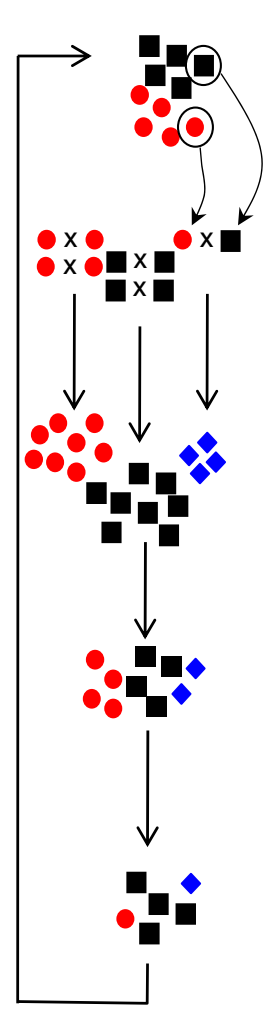

1. Mating

a) Randomly select two individuals (encounter event)

b) Mate with probability: $A A \times A A, A a \times A a$, aa $x$ aa: 1 AA $x$ aa: $\quad n$ $\mathrm{AA} \times \mathrm{Aa}$, aa $\times \mathrm{Aa}: \quad(1+\boldsymbol{n}) / 2$

c) Mating function: Promiscuous Offspring/mating pair: 1

Return to mating pool: yes

Repeat until: $\quad 2 K \rho$ encounter events
Monogamous

$\rho$

no

all mated
2. Inheritance

Parent alleles Offspring allele inherited probability

$\mathrm{A} / \mathrm{a} \mathrm{X}_{1} \quad \mathrm{X}_{1} \mathrm{Y}_{1} \quad(1-R) / 2$

$\left(\begin{array}{lll}X_{1} Y_{1} \\ X_{2} Y_{2}\end{array} \quad X_{2} Y_{2} \quad(1-R) / 2\right.$

$\mathrm{X}_{2} \mathrm{Y}_{1} \quad \boldsymbol{R} / 2$

3. Density-dependence: genotype probability survive

$$
\begin{array}{ll}
\text { AA } & \left(1+\frac{\rho_{A A} / 2-1}{(1+s) K \rho_{A A} / 2}\left(N_{A A}^{\prime}+\frac{1+s}{2} N_{A a}^{\prime}+s N_{a a}^{\prime}\right)\right)^{-1} \\
\text { Aa } & \left(1+\frac{\rho_{A a} / 2-1}{(1+s) K \rho_{A a} / 2}\left(N_{A a}^{\prime}+\frac{1+s}{2}\left(N_{A A}^{\prime}+N_{a a}^{\prime}\right)\right)\right)^{-1} \\
\text { aa } & \left(1+\frac{\rho_{a a} / 2-1}{(1+s) K \rho_{a a} / 2}\left(N_{a a}^{\prime}+\frac{1+s}{2} N_{A a}^{\prime}+s N_{A A}^{\prime}\right)\right)^{-1}
\end{array}
$$

4. Density-independent survivorship/selection: probability survive $=w_{i j} v_{k l}$

\begin{tabular}{ll} 
Locus 1 genotype & fitness \\
\hline AA & $w_{A A}=1$ \\
Aa & $w_{A a}=\boldsymbol{h}$ \\
aa & $w_{a a}=1$
\end{tabular}

$\begin{array}{cl}\text { Locus 2 genotype } & \frac{\text { fitness }}{v_{B B}=\boldsymbol{f}} \\ \mathrm{BB} & v_{B b}=(\boldsymbol{f}+\boldsymbol{g}) / 2 \\ \mathrm{Bb} & v_{b b}=\boldsymbol{g}\end{array}$

mating, and an intermediate value indicates imperfect assortative mating.

In the "promiscuous mating function," individuals are sampled with replacement (i.e., they return to the mating pool after the encounter, regardless of whether mating occurred), and the selection process repeats for a set number of encounters (a probabilistic version of O'Donald 1960). Therefore, the number of mating events for a given individual is stochastic and variable across the population. In the "monogamous mating function," if individuals successfully mate, they are not returned to the mating pool and the selection process repeats until all individuals are in a designated mating pair (similar to Gavrilets and Boake 1998 with infinite encounter events). Therefore, all individuals mate once per generation with one other individual (unless there is an odd number of individuals, in which case one individual does not mate). As a result, mating success depends on relative population densities more in the promiscuous than the monogamous mating function. Difficulty with finding mates at low densities is frequently invoked in Allee effects (Courchamp et al. 1999; Stephens et al. 1999); here we do not explicitly incorporate Allee effects but rather incorporate mating dynamics that might drive them.

Then, in the monogamous mating function, each mating pair produces $\rho$ offspring; in the promiscu- ous mating function, each mating event produces one offspring. In order to have a similar number of offspring per individual on average in both mating functions at equilibrium, the total number of encounters in the promiscuous mating function depends on $\rho$. Specifically, the carrying capacity for each species $K$ indicates the expected number of individuals of each species when stable coexistence occurs. Given strong assortative mating (a low value for $n$ ), half of the encounters are unlikely to end up in successful mating, and $2 K$ encounter events are necessary for an individual to have one successful encounter on average. Therefore, $2 K \rho$ encounter events lead to an average production of $\rho / 2$ offspring per individual ( $\rho$ per pair). While the total number of encounters necessary for $\rho / 2$ offspring per individual will depart from this value if densities deviate from the carrying capacity or under weak assortative mating, the number of encounter event remains constant, independent of these values, under the assumption that it is representative of the length of the mating season rather than these conditions.

The deterministic version most closely resembles the promiscuous mating function: The proportion of each mating type (parental genotype pair) is the normalized product of the encounter probability and the probability of mating given an encounter. As with the 
stochastic version, the encounter probability is the product of the proportion of individuals with each of the two genotypes, and the probability of mating given an encounter is 1 for individuals of the same species, $n$ for individuals of different species, and $(1+n) / 2$ for hybrids and homozygotes for either species. We then multiply the proportion of each mating type by the total population size and the average reproduction of $\rho / 2$ offspring per individual ( $\rho$ per pair) to arrive at the number of offspring for each mating type.

\section{Inheritance}

To determine the genotype for each offspring, in each parent recombination between the two loci occurs with probability $R$ (zero for no recombination, 0.5 for unlinked loci). Then each parent passes along one of its two subsequent gametes to the offspring with equal likelihood. This representation follows the classic population genetic model by Lewontin and Kojima (1960). In the deterministic version, these probabilities determine the proportion of offspring with each genotype.

\section{Density-dependent survivorship}

To constrain population growth, the model includes density dependence with carrying capacity for each species $K$. Whether or not the two species affect the density-dependent dynamics of each other depends on whether they engage in interference competition and/or compete for the same limiting resources. Let $0 \leq s \leq 1$ represent the overall strength of interspecific competition, or the density-dependent effect an individual of the other species relative to an individual of its own species: $s=0$ for no interspecific competition (the default), $s=1$ for equivalent interspecific and intraspecific competition, and $s$ takes on an intermediate value for partial overlap in resource use. Again, hybrids are intermediates, where the competitive effect of hybrids on homozygotes (and vice versa) is $(1+s) / 2$ and the competitive effect of hybrids on each other is 1 . Therefore, given the number of individuals of each species $i j(i, j \in\{\mathrm{a}, \mathrm{A}\})$ at time $t$ after reproduction $N_{i j, t}^{\prime}$, the realized number of individuals $\tilde{N}_{i j, t}^{\prime}$ that affect the density-dependent survivorship is $\tilde{N}_{\mathrm{AA}, t}^{\prime}=N_{\mathrm{AA}, t}^{\prime}+$ $s N_{\mathrm{aa}, t}^{\prime}+(1+s) N_{\mathrm{Aa}, t}^{\prime} / 2$ for species 1 individuals, $\tilde{N}_{\mathrm{aa}, t}^{\prime}=$ $N_{\mathrm{aa}, t}^{\prime}+s N_{\mathrm{AA}, t}^{\prime}+(1+s) N_{\mathrm{Aa}, t}^{\prime} / 2$ for species 2 individuals, and $\tilde{N}_{\mathrm{Aa}, t}^{\prime}=N_{\mathrm{Aa}, t}^{\prime}+(1+s)\left(N_{\mathrm{AA}, t}^{\prime}+N_{\mathrm{aa}, t}^{\prime}\right) / 2$ for hybrid individuals.

This density-dependent survivorship derives from the classic Beverton-Holt model $N_{t+1}=r N_{t} /(1+$ $\alpha N_{t}$ ), where $\alpha$ determines the strength of density dependence and $r$ is the per-capita reproduction (half of the per-pair reproduction, e.g., $\rho / 2$ given the monogamous mating function). Specifically, each of the individuals produced in the reproduction step described above (reflective of the $r N_{t}$ term) survives densitydependent mortality with probability $\left(1+\alpha_{i j} \tilde{N}_{i j, t}^{\prime}\right)^{-1}$. In order to express this in terms of a constant carrying capacity $K$ for each species, we let $\alpha_{i j}=\left(\rho_{i j} / 2-\right.$ $1) /\left((1+s) K \rho_{i j} / 2\right)$, where $\rho_{i j}=\rho$ for all species given the monogamous mating function and $\rho_{i j}$ is calculated directly from the number of offspring per pair within each species each generation given the promiscuous mating function (where, for $\alpha_{i j}$ to remain positive, $\rho_{i j} / 2$ takes a minimum values of 1.000001 when there are fewer than one offspring per adult of species $i j$ ). These probabilities (with $\rho_{i j} / 2$ calculated from the perindividual reproductive output of each species type) determine the proportion of individuals of each species surviving in the deterministic version.

\section{Selection (density-independent survivorship)}

Selection based on fitness at both alleles then occurs through density-independent mortality (hard selection). Specifically, given fitness at the speciesidentifying locus $w_{i j}(i, j \in\{\mathrm{A}, \mathrm{a}\})$ and fitness at the environmental condition-dependent locus $v_{k l}$ $(k, l \in\{\mathrm{B}, \mathrm{b}\})$, individuals survive with probability $w_{i j} \cdot v_{k l}$. Analogously, in the deterministic version, the proportion of individuals with each genotype surviving the selection step is $w_{i j} \cdot v_{k l}$. Given that the fitness values represent the probability of surviving density-independent mortality, their values must be between 0 and 1 .

We use disruptive selection to model hybrid inferiority at the first locus, and we implement environmental change with directional selection against the $\mathrm{b}$ allele at the second locus. Because the same locus that determines mating assortment influences disruptive selection via hybrid inferiority, this model falls into the class of "magic trait" models (Gavrilets 2004). Specifically, to represent general hybrid inferiority, the fitness of a heterozygote at the first locus (Aa) is lower than homozygotes, with equal fitness for the two homozygotes (AA for species 1 and aa for species 2), i.e., a relative hybrid fitness of $w_{\mathrm{Aa}}=h<1$ compared to the homozygote fitness of $w_{\mathrm{AA}}=w_{\mathrm{aa}}=1$. For the second locus, we let the fitness for BB homozygotes be $v_{\mathrm{BB}}=f=1$, the relative fitness for bb homozygotes be $v_{\mathrm{bb}}=g$, and heterozygotes to have intermediate fitness, $v_{\mathrm{Bb}}=(f+g) / 2$, i.e., we ignore dominance. Before the environmental shift, the fitness is equal for homozy- 
(a) $g=1$

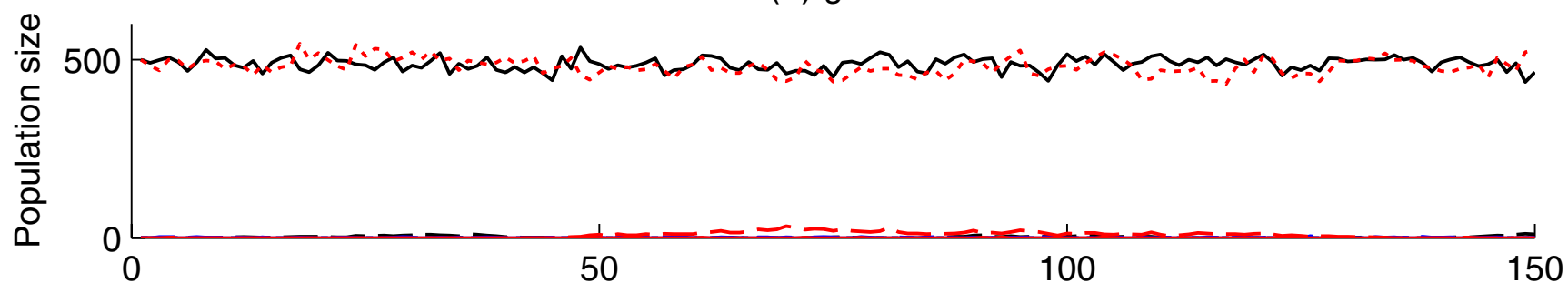

(b) $g=0.7$

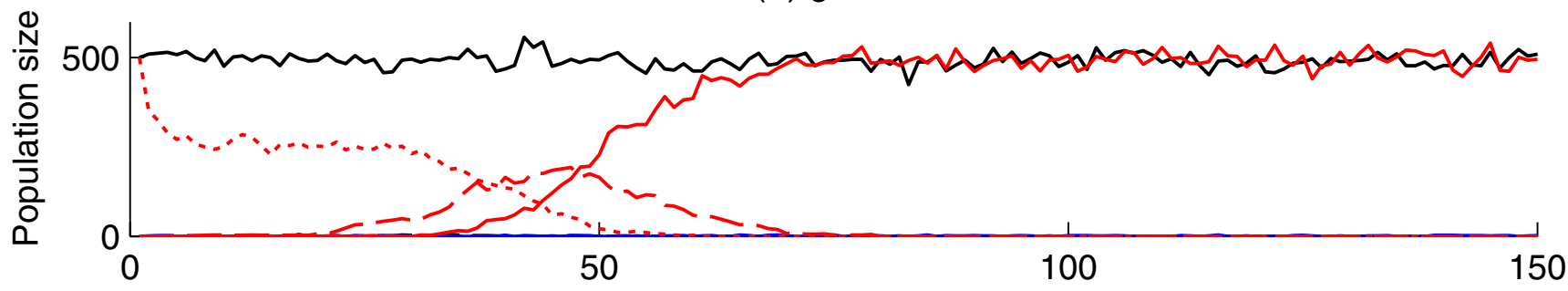

(c) $g=0.3$

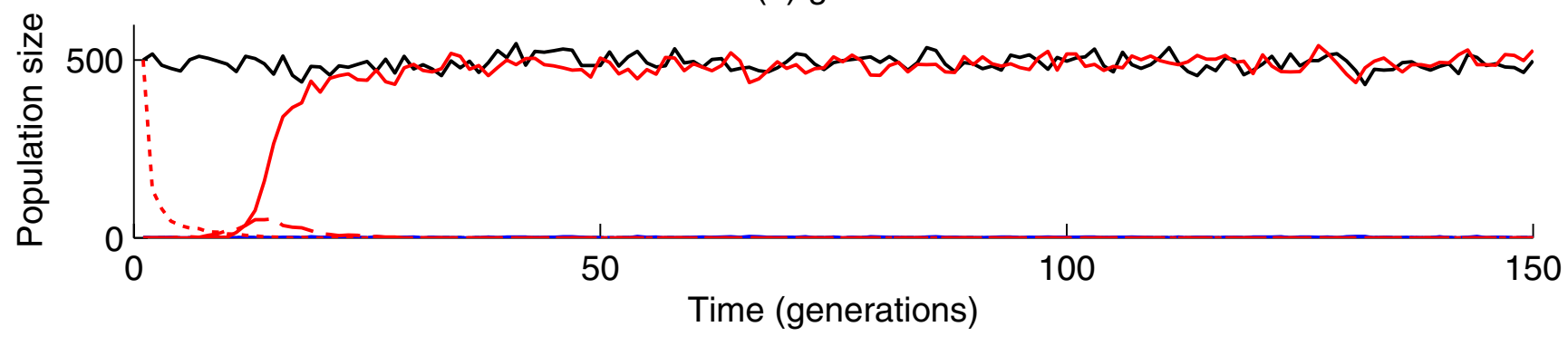

Fig. 2 Sample time series of simulation runs for default parameter values (Table 1) and the monogamous mating function. Here and in all subsequent figures, colors distinguish the species, and line and symbol types distinguish genotypes at the second locus: black lines represent species 1 (AA individuals), red lines represent species 2 (aa individuals), and blue lines represent hybrids (Aa individuals); solid lines represent homozygotes for the advantageous allele at the second locus (BB individuals), dotted lines represent homozygotes for the disadvantageous allele at the second locus after environmental change (bb individuals), and dashed lines represent heterozygotes at the second locus $(\mathrm{Bb}$ individuals). a The maintenance of AABB (solid black line) and aabb (dotted red line) genotypes before any change in fitness at the second locus $(g=1)$. b, c Introgression leading to the eventual coexistence of the aaBB genotype (solid red line) along with the $\mathrm{AABB}$ genotype for different values for the fitness at the second locus after environmental change $(g=0.7$ in $\mathbf{b}$ and $g=0.3$ in $\mathbf{c})$

Table 1 Parameters and default values

\begin{tabular}{lll}
\hline Parameter & Description & Default value \\
\hline$n$ & Probability mate other species given encounter (determines degree of assortative mating) & 0.01 \\
$h$ & Relative hybrid fitness: survivorship of individuals with genotype Aa at the first locus & 0.1 \\
$f$ & Survivorship of individuals with the advantageous genotype (BB) at the second locus & 1 \\
$g$ & Survivorship of individuals with the disadvantageous genotype (bb) at the second locus & 0.7 \\
& after the environmental shift & 0.2 \\
$K$ & Probability of recombination between the two loci & 500 individuals \\
$s$ & Carrying capacity for each species & 0 \\
$\rho$ & Strength of interspecific competition & 6 \\
$t_{f}$ & Average number of offspring per pair & 150 generations \\
\hline
\end{tabular}


(a)

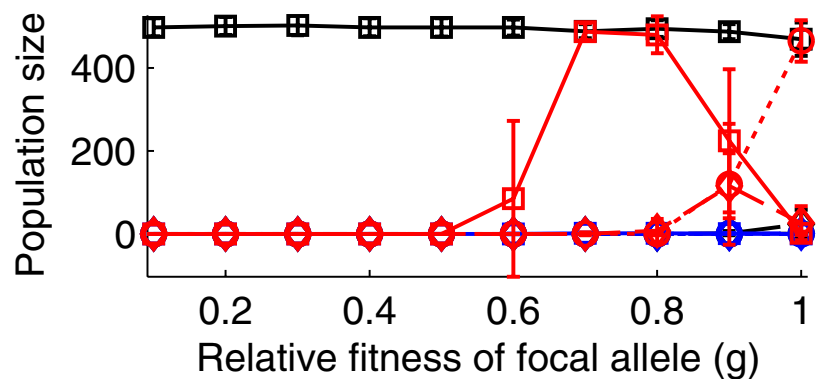

(c)

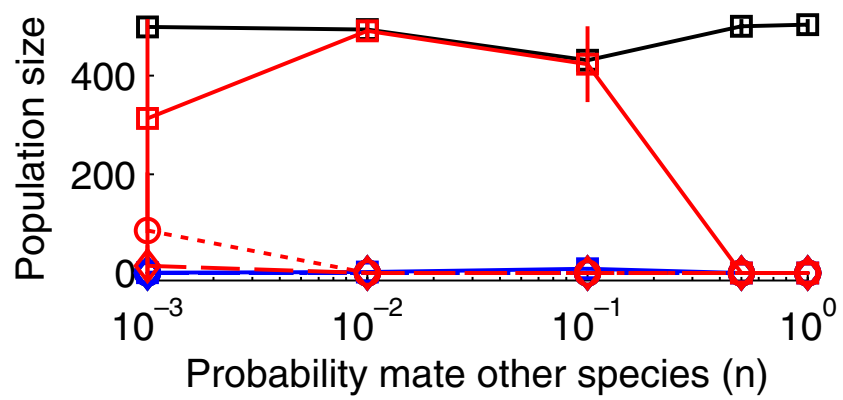

(e)

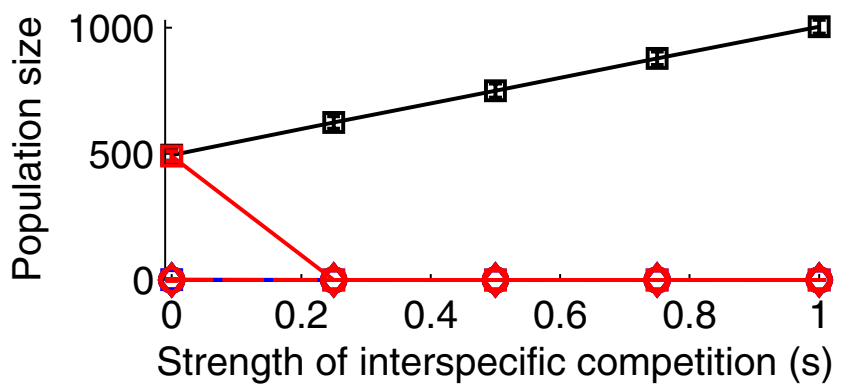

Fig. 3 Effects of changing each parameter given the promiscuous mating function in the stochastic model: a the relative fitness of the disadvantageous allele (allele $b$ at the second locus) after the environmental shift $v_{\mathrm{bb}}=g, \mathbf{b}$ the relative hybrid fitness $w_{\mathrm{Aa}}=h$, c the probability of interspecific mating given an encounter $n$ (stronger assortative mating occurs with lower probability; note the log scale on the $x$-axis), $\mathbf{d}$ the probability of recombination $R$, e the strength of interspecific competition $s$, and $\mathbf{f}$ the carrying capacity $K$ (note the $y$-axis scaled to $K$ ). Here and in all subsequent figures, colors distinguish the species, and line and symbol types distinguish genotypes at the second locus: black lines represent species 1 (AA individuals), red lines represent species 2 (aa individuals), and blue lines represent hybrids

gotes of each genotype $(g=1)$. Then the environment shifts such that homozygotes for allele $b$ have lower fitness $(g<1)$.

The goal of the model is to test whether introgression at the second locus rescues the second species from extinction following the change in fitness from $g=1$ to $g<1$. This is basically a question of the maintenance of genetic variation at the first locus $(\mathrm{A}, \mathrm{a})$ under disruptive selection, with assortative mating acting analo- (b)

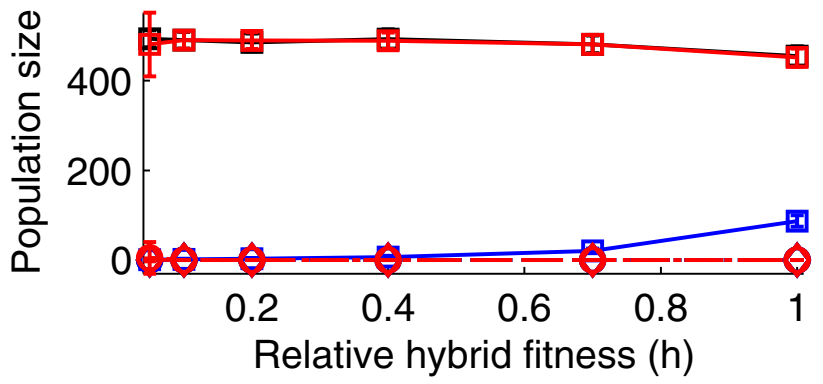

(d)

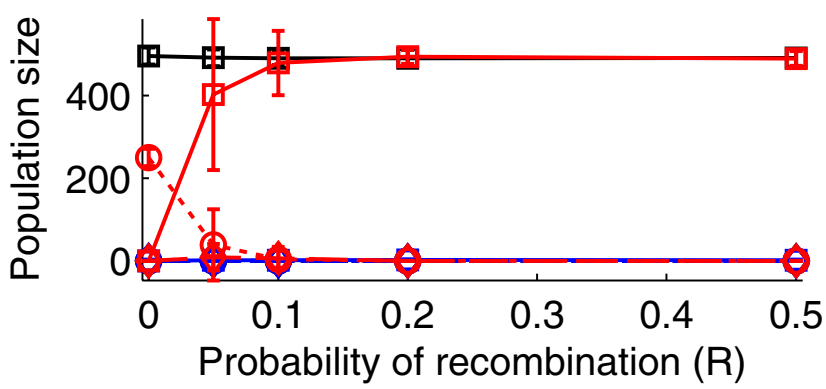

(f)

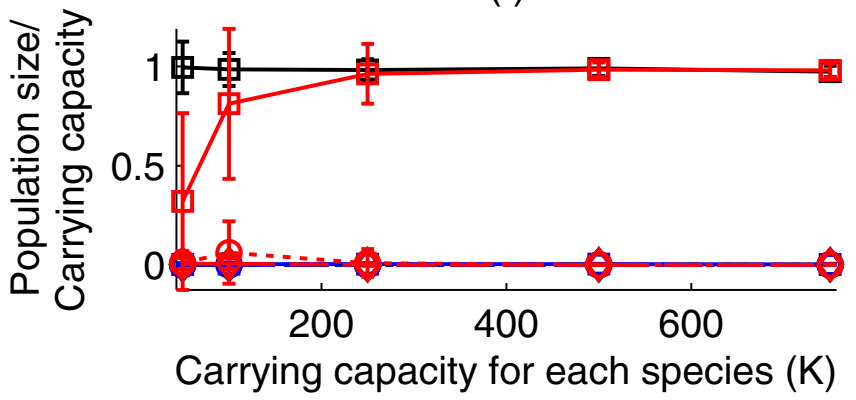

(Aa individuals); solid lines with squares represent homozygotes for the advantageous allele at the second locus (BB individuals), dotted lines with circles represent homozygotes for the disadvantageous allele at the second locus after environmental change (bb individuals), and dashed lines with diamonds represent heterozygotes at the second locus ( $\mathrm{Bb}$ individuals); error bars indicate standard deviations. Therefore, solid black lines with squares (AABB) and solid red lines with squares (aaBB) at their carrying capacities represent successful introgression. In addition, a solid red line below carrying capacity with large error bars typically reflects a mix of extinction $\left(n_{\mathrm{aa}}=0\right)$ and successful introgression $\left(n_{\text {aa }} \approx K\right)$ across different simulation runs

gously to the separation in space with "weak coupling" via migration that can also maintain polymorphisms under disruptive selection (Karlin and McGregor 1972; Svirezhev 1968). However, central to our interest in introgressive hybridization in response to environmental change is, more specifically, whether or not the outcome is persistent $\mathrm{AABB}$ and aaBB populations given the starting point of $\mathrm{AABB}$ and aabb populations and a drop in fitness for the $b$ allele. 


\section{Model implementation}

Implementing the model as a stochastic individualbased simulation allows for the incorporation of demographic stochasticity, including its role in encounter probabilities and extinction likelihood. Each simulation starts with each of the two species at its carrying capacity $K$ and homozygous for different alleles at each locus, i.e., $K$ AABB individuals (species 1 ) and $K$ aabb individuals (species 2). Then the simulations iterate the mating, inheritance, density-dependent survivorship, and selection steps described above for $t_{f}$ generations (where $t_{f}$ is beyond the amount of time necessary for the populations to reach quasi-equilibrium in test simulations with the default parameter values). Successful introgression occurs when the population consists of $\sim K$ AABB individuals and $\sim K$ aaBB individuals at time $t_{f}$.

First, we present a sample time series (Fig. 2) to illustrate the basic dynamics of the stochastic model and to confirm that the default parameter values (Table 1: high degree of assortative mating, low hybrid fitness, intermediate environmental change, intermediate recombination, and no interspecific competition) lead to the maintenance of separate AABB and aabb genotypes before the environmental change and intro- (a)

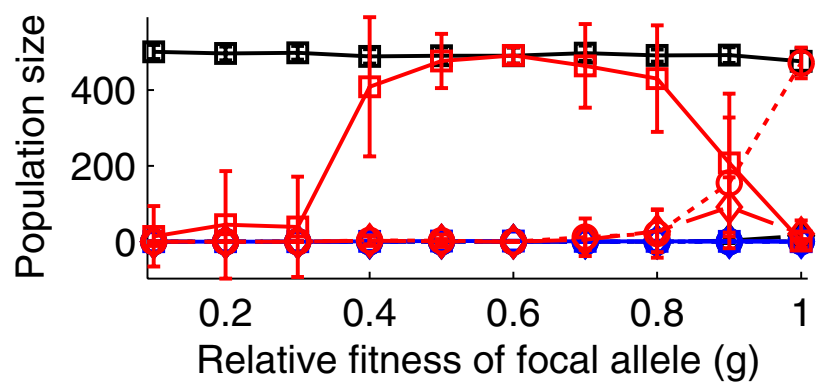

(c)

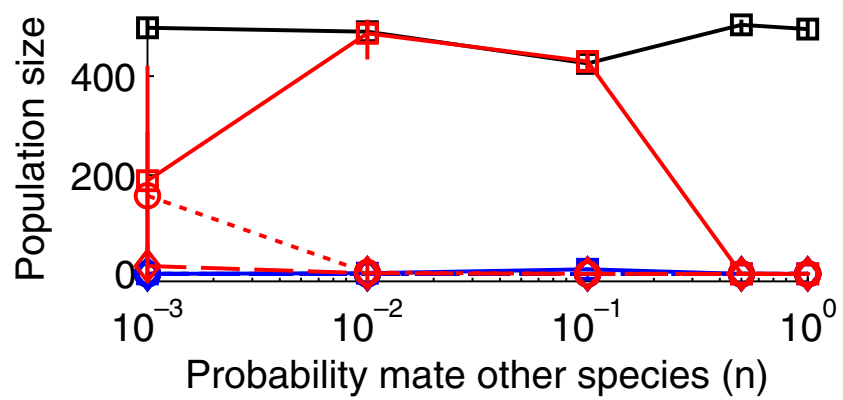

(e)

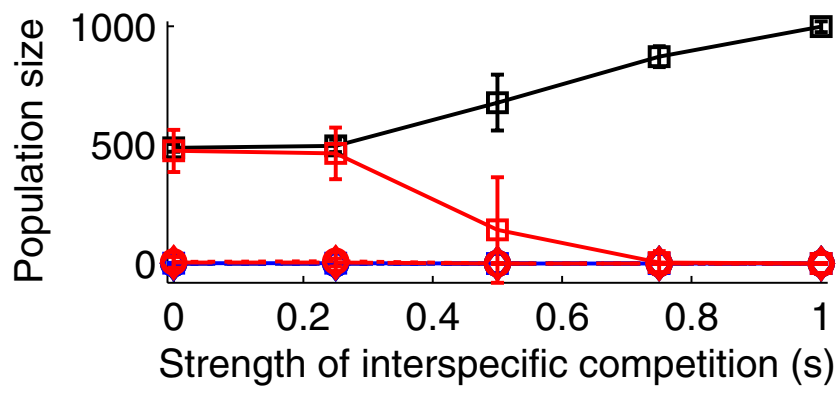

Fig. 4 Effects of changing each parameter given the monogamous mating function and demographic stochasticity: a the relative fitness of the disadvantageous allele (allele $b$ at the second locus) after the environmental shift $v_{\mathrm{bb}}=g, \mathbf{b}$ the relative hybrid fitness $w_{\mathrm{Aa}}=h$, c the probability of interspecific mating given an encounter $n$ (stronger assortative mating occurs with lower (b)

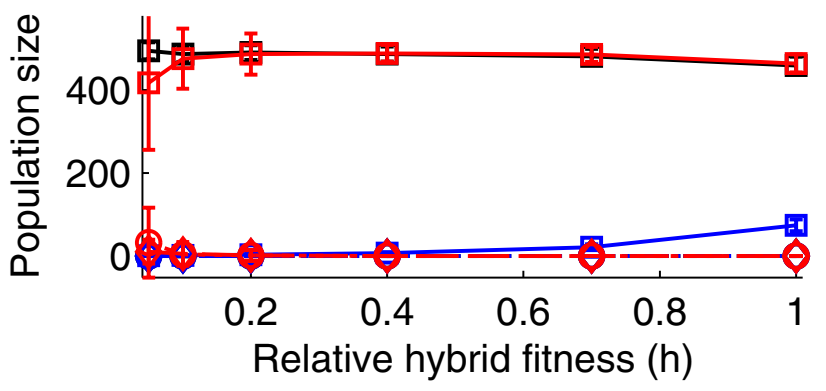

(d)

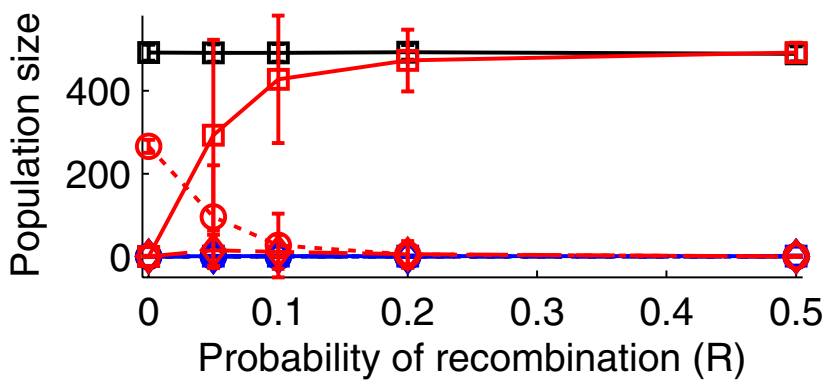

(f)

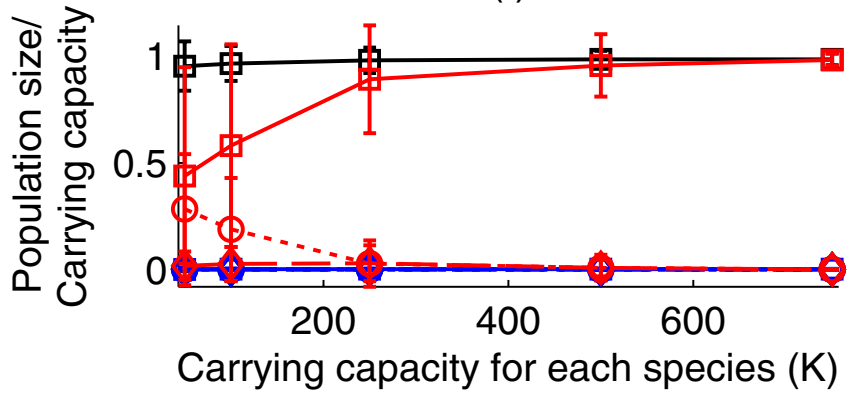

probability; note the log scale on the $x$-axis), $\mathbf{d}$ the probability of recombination $R$, e the strength of interspecific competition $s$, and $\mathbf{f}$ the carrying capacity $K$ (note the $y$-axis scaled to $K$ ). All colors, lines, and symbols are as in Fig. 3, where solid black lines with squares $(\mathrm{A} A \mathrm{BB})$ and solid red lines with squares $(\mathrm{a} \mathrm{BB})$ at their carrying capacities represent successful introgression 
gression of the B gene into species 2 after the environmental change. Then we vary each parameter value in order to determine its effect on the likelihood of introgression given each mating function (Figs. 3 and 4, with the equivalent results without environmental change, or $g=1$, in ESM B to determine the effect of these parameter values on the maintenance of separate species). We run the simulation for each combination of parameter values 100 times and present the mean and standard deviation of the number of individuals with each genotype at time $t_{f}$ across all runs. Finally, we compare these results from the stochastic model to the analogous deterministic model in order to determine the role of demographic stochasticity (Fig. 5 and ESM C).

(a)

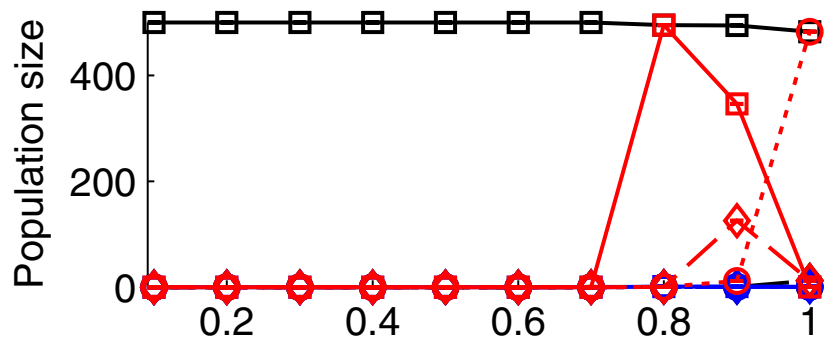

Relative fitness of focal allele $(\mathrm{g})$

(c)

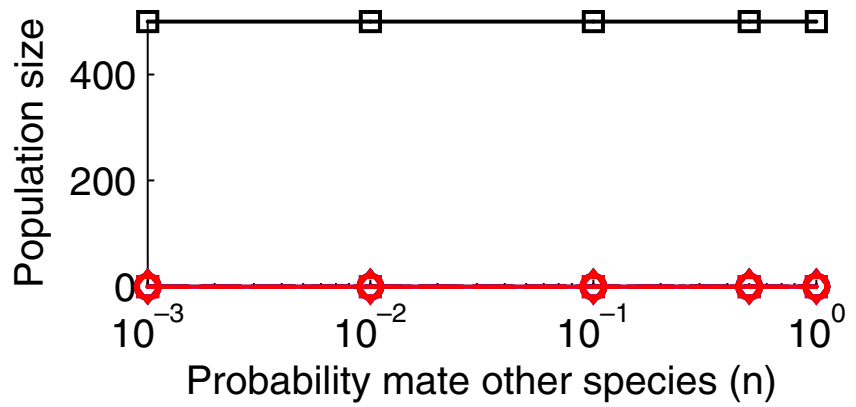

(e)

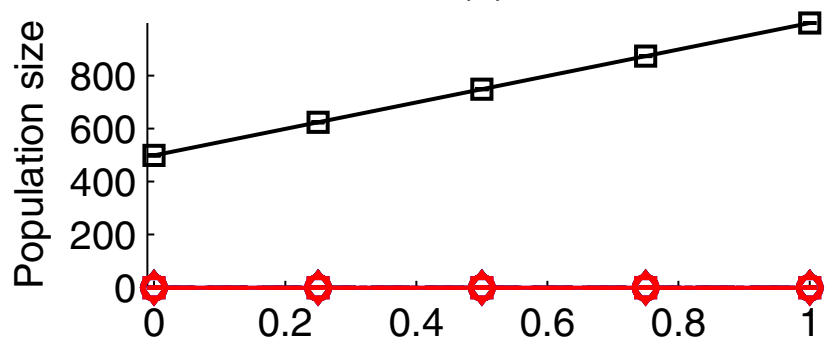

Strength of interspecific competition (s)

Fig. 5 Results of the deterministic model (no demographic stochasticity, otherwise analogous to the promiscuous mating function simulations): population sizes at 150 generations for different

\section{Results}

Sample time series

In the sample time series with the default parameter values, before the environmental change (when $g=1$, Fig. 2a), assortative mating and hybrid inferiority maintain separate species with rare hybridization. After the environmental change (when $g<1$, Fig. $2 b$, c), introgression at the second allele can occur. In trial runs, the time necessary for introgression to occur decreases with increasing number of offspring per individual $\rho / 2$ (i.e., higher reproductive output means faster evolutionary response; results not shown) and with decreasing relative fitness at the disadvantageous allele after envi-

(b)

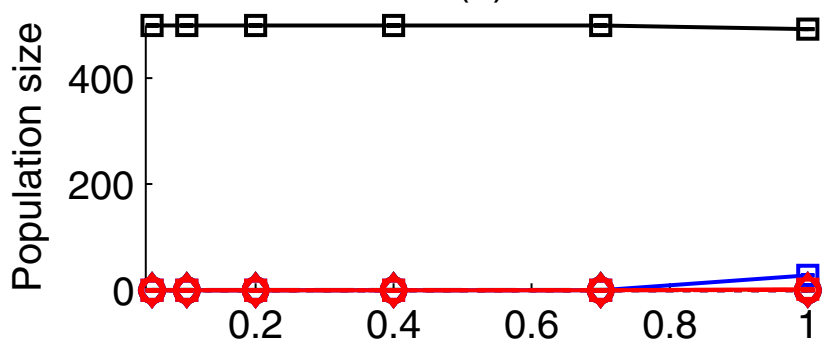

Relative hybrid fitness ( $h$ )

(d)

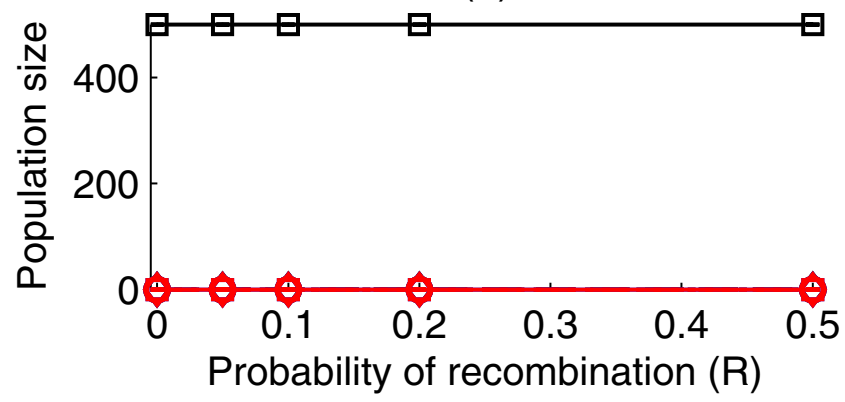

(f)

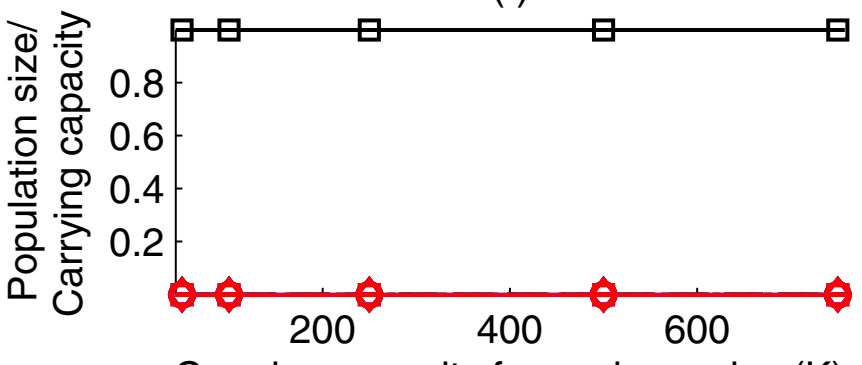

Carrying capacity for each species $(\mathrm{K})$

parameter values, equivalent to the ranges explored in Figs. 3 and 4. All colors, lines, and symbols are as in Fig. 3 
ronmental change $g$ (i.e., greater environmental change means faster evolutionary response; Fig. 2b, c).

\section{Effect of each parameter and mating system}

In stepping through the effect of each parameter value, we focus on the values necessary for successful introgression to occur. Introgression occurs at intermediate values for the relative fitness of the disadvantageous allele (allele $b$ at the second locus) after the environmental shift $\left(v_{b b}=g\right.$; Figs. 3a and $\left.4 \mathrm{a}\right)$. When $g=1$, there is no selection for introgression to occur, and assortative mating and hybrid inferiority at the first locus maintain the separate AABB and aabb populations (black solid and red dotted lines, respectively). For relative fitness close to 1 , weak selection against the disadvantageous allele leads to slower introgression and a greater role of stochasticity and therefore highly variable populations of aabb, aaBb, and aaBB individuals (red dotted, dashed, and solid lines, respectively). At low values of relative fitness, the overall lower survivorship of hybrids $\left(w_{\mathrm{Aa}} v_{\mathrm{Bb}}=h(1+g) / 2\right)$ leads to a greater potential for species 2 collapse rather than backcrossing and introgression. This effect is stronger for the promiscuous mating function (Fig. 3a) than the monogamous mating function (Fig. 4a) because of the potential for the surviving hybrids to have fewer successful matings by random chance with promiscuous mating (as opposed to the monogamous mating function, where all individuals mate). Here and elsewhere, a mix of extinction $\left(N_{\mathrm{aa}}=0\right)$ and successful introgression $\left(N_{\mathrm{aa}} \approx K\right)$ across different simulation runs drives the variation illustrated by the error bars in the end aaBB population sizes (solid red squares when all other red points, representing the $\mathrm{aaBb}$ and aabb population sizes, are zero).

Introgression occurs across a broad range of intermediate values for the relative hybrid fitness $\left(w_{\mathrm{Aa}}=h\right.$, where a greater hybrid inferiority occurs with lower hybrid fitness; Figs. $3 b$ and $4 b$ ). At low values, the species 2 population with the advantageous allele (aaBB genotype, solid red lines with squares) is lower on average and has higher variability, with higher sizes for the population with the disadvantageous allele. This result, which is more prominent with the monogamous mating function (Fig. 4b) than in the promiscuous mating function (Fig. 3b), arises because the lower hybrid survivorship leads to less of a chance of introgression occurring and therefore more of a chance of collapse for species 2 , as well as slower introgression when it does occur. As hybrid inferiority vanishes (relative hybrid fitness increases to 1 ), the equilibrium includes a stable dis- tribution of species 1 , species 2 , and hybrids all with the advantageous allele (AABB, aaBB, and AaBB, in the black, red, and blue solid lines with squares, respectively) rather than a maintenance of separate species. Separate species are also not maintained at high values for hybrid fitness before the environmental change ( $g=1$; Figs. B.1a and B.2a in ESM B).

Introgression also occurs at intermediate values of the probability of interspecific mating given an encounter ( $n$; stronger assortative mating occurs with lower probability; Figs. $3 \mathrm{c}$ and $4 \mathrm{c}$ ). At low values, the species 2 population with the advantageous allele (aaBB individuals, solid red lines with squares) is lower on average and has higher variability, with higher population sizes for the population with the disadvantageous allele (aabb individuals, dotted red lines with circles). This result, which is more pronounced with the monogamous mating function (Fig. 4c) than with the promiscuous mating function (Fig. 3c), arises because the fewer hybridization events lead to less of a chance of introgression occurring and therefore more of a chance of collapse of the second species, as well as slower introgression when it does occur. At high values, species 2 collapses rather than introgression occurring due to weaker assortative mating causing hybridization to occur more frequently than backcrossing in conjunction with hybrid inferiority and density dependence (Aa individuals experience a greater densitydependent effect from the AA population than aa individuals). Weaker assortative mating also leads to frequent hybridization rather than the maintenance of separate species without environmental change ( $g=1$; Figs. B.1b and B.2b in ESM B).

The potential for introgression increases with increasing probability of recombination ( $R$; Figs. $3 \mathrm{~d}$ and $4 d)$. This intuitive result arises because of the increased rate at which species 1 indicator (a) can become associated with the newly favorable genotype at the second locus (B) on the same allele through recombination.

With increasing strength of interspecific competition ( $s$; Figs. $3 \mathrm{e}$ and $4 \mathrm{e}$ ), the probability of introgression declines, and the probability of species 2 collapse increases because of the lower density-dependent survivorship for the initially disadvantaged species 2 individuals. This effect is stronger in simulations with the promiscuous mating function (Fig. 3e) than in the monogamous mating function (Fig. 4e) because of the greater stochasticity in mating success. With decreasing carrying capacity ( $K$; Figs. $3 \mathrm{f}$ and $4 \mathrm{f}$ ), the population sizes are more variable and introgression is less likely because of the greater demographic stochasticity with fewer individuals. 


\section{Deterministic model}

In the deterministic version of the model (Fig. 5), introgression occurs over a much narrower range of parameter values as compared to the model with demographic stochasticity (Figs. 3 and 4). Rather than introgression, species 2 collapses in all cases of the deterministic model except when there is little or no change in fitness at the second allele $(g \geq 0.8$; Fig. 5a). This outcome is due to the initialization of each simulation with all $\mathrm{AABB}$ and aabb individuals: After environmental change $(g<1)$, equilibria with $\mathrm{AABB}$ alone, aaBB alone, and $\mathrm{AABB}$ and $\mathrm{a} \mathrm{BB}$ at equal densities are possible but depend on initial conditions. In particular, for coexistence of $\mathrm{AABB}$ and $\mathrm{aaBB}$ to occur, both must start with population sizes above a threshold density (see ESM C for demonstration). For the simulations in Fig. 5, given that all species 2 (aa) individuals are initially associated with the disadvantageous allele (bb), in most cases the introgressed individuals $(\mathrm{aaBb}$ and, eventually, aaBB) do not reach the threshold density necessary for their eventual coexistence with species 1 (AABB).

\section{Discussion}

Here we use a population genetic model with demographic stochasticity to determine when introgressive hybridization can play a role in adaptive response to environmental change. Simulation results indicate that both having separate sub-species before an environmental shift (ESM B) and introgression after the shift depends on a balance of assortative mating strength (Figs. 3c and 4c), hybrid inferiority (Figs. 3b and 4b), and the change in fitness in the focal allele (Figs. 3a and 4a). Below we explore the implications of this balance in terms of the broader theoretical context and potential species characteristics that indicate a greater likelihood of introgressive hybridization playing a role in adaptation to environmental shifts.

The amount of environmental change to which a population can adapt

In the model presented here, successful introgressive hybridization requires an intermediate drop in relative fitness at the locus negatively affected by environmental change (Figs. 3a and 4a). With too much environmental change (lower fitness), the lower survivorship of the affected species leads to extinction, precluding successful introgression; with too little fitness change (higher fitness), weaker selection leads to slower genetic dynamics. This result parallels previous models that suggest that the amount of sudden environmental change populations can adapt to is limited (Gomulkiewicz and Holt 1995; Orr and Unckless 2008). However, inclusion of additional genetic diversity from hybridization, as shown here, does have the potential to expand this capacity.

In particular, the affected species can respond to a much wider range of environmental change given the monogamous mating function (Fig. 4a) as compared to the promiscuous mating function (Fig. 3a). This result arises from the lower stochasticity in mating likelihood in our monogamous mating function, where individuals eventually find mates if they are available and every individual mates once. In contrast, in our promiscuous mating function, some individuals may mate many times and others not at all, depending on encounter events, so rare groups (e.g., hybrids, the species affected by environmental change) might fail to mate. In other words, the important driving factor for the different results for our two mating systems is not the degree of monogamy per se; rather, it is whether the mating system incurs any cost to assortative mating through reduced mating success when the preferred type is rare (lower reproductive assurance). This importance of the cost to assortative mating occurs in models of assortative mating-driven speciation as well (Gavrilets 2004).

Therefore, introgressive hybridization in response to environmental change is more likely for species with mating system characteristics that reduce stochasticity in mating likelihood. In addition to monogamy itself, such characteristics include mechanisms for finding preferred individuals in a crowded mating field, such as birdsong (Grant and Grant 2008) and assortment in time (Bearhop et al. 2005), instead of depending on random encounters. In addition to finding preferred individuals, mating success depends on eventual hybridization if few such individuals are available, i.e., a frequency-dependent mating dynamics where, as fewer mate choices are available, preference standards decline, and hybridization increases (similar to the model by Chan and Levin 2005). Empirical evidence supports the potential for mating preference to be relative to the available mating pool (rather than absolute) or to decline over the course of a mating season for many organisms (Wirtz 1999).

The balance of assortative mating and hybridization

Intermediate assortative mating (low, but not too low, values for the probability of mating another species 
given an encounter; Figs. 3c and 4c) is necessary for successful introgressive hybridization rather than extinction of the species affected by environmental change. Too much assortment leads to too little hybridization. Too little assortment leads to too much hybridization rather than backcrossing and then the loss of the affected species due to hybrid inferiority and densitydependent mortality given partial competition between hybrids and the unaffected species. The degree of hybrid inferiority is less critical to successful introgression than the degree of assortative mating as long as it is above a fairly low threshold (Figs. 3b and 4b), although it does substantially affect the maintenance of separate species before the environmental change (Figs. B.1a and B.2a in ESM B; consistent with the existing theory of speciation given disruptive selection and assortative mating, Gavrilets 2004). The stronger effect of assortative mating than hybrid inferiority here echos the more rapid introgression with pre-zygotic reproductive isolation as compared to post-zygotic isolation given frequency-dependent mating in the model by Chan and Levin (2005). Overall, these results suggest a greater importance of assortative mating than hybrid inferiority in determining whether introgressive hybridization in response to environmental change is feasible. The range of assortative mating where introgressive hybridization can occur has the potential to apply to a number of organisms given the wide variety of taxa where introgressive hybridization has been observed (Anderson 1948; Arnold et al. 2008; Arnold and Martin 2010).

\section{Genetic structure and model assumptions}

High recombination between the two loci enhances the potential for introgression (Figs. 3d and 4d) because of the weaker association between the gene affected by environmental change and the gene that identifies the species in the simplified representation here. In the extreme case of perfect linkage (i.e., no recombination), introgression does not occur, which parallels the results by Proulx (1999) where assortative mating based on the same locus that determines local fitness reduces the theoretical potential for adaptation to a novel environment. Weaker association between the species identifier and the traits affected by environmental change might particularly apply to the case where the traits that influence assortative mating are non-genetically transmitted. For example, bird song, the trait that determines assortment for the Darwin's finch example of introgression described in the "Introduction," is transmitted through sexual imprinting (Grant and Grant 2002). Given its capacity to affect species iden- tification and assortative mating, the effect of sexual imprinting on introgression in response to environmental change is a topic well worth future theoretical exploration.

The two-locus, biallelic additive model used here as the simplest possible representation of the relevant dynamics ignores the reality that species-identifying and fitness-determining traits likely depend on complex gene structures. Additional genetic complexity, such as multiple major and minor loci influencing a particular trait, can significantly influence the potential for and rate of evolution in response to environmental change (Gomulkiewicz et al. 2010). While more interchangeable loci or more loci with small effects might increase the potential for adaptation to environmental change (Orr and Unckless 2008; Boulding and Hay 2001), having multiple genes influencing a particular trait might reduce the likelihood of introgression because of the lower likelihood of combinations of genes moving together (Barton 2001). Here we also assume complete heritability; quantitative genetic models indicate that greater heritability increases the likelihood of adaptation to environmental shifts (Boulding and Hay 2001; Gomulkiewicz and Holt 1995).

In addition to Mendelian traits, we assume biparental nuclear inheritance. Given assortative mating, introgression might occur more rapidly with paternally or maternally inherited loci (e.g., mitochondrial DNA; Chan and Levin 2005). Finally, we ignore mutation, but mutation is unlikely to contribute to response to environmental change when considering demographic stochasticity given the large probability of losing rare mutations by chance (depending on whether the mutations are deleterious or neutral before the environmental change; Orr and Unckless 2008). Overall, results from models of adaptive response to environmental change without hybridization (Gomulkiewicz et al. 2010; Gomulkiewicz and Holt 1995; Boulding and Hay 2001; Orr and Unckless 2008), or of introgression without environmental change (Barton 2001; Chan and Levin 2005), suggest that incorporating additional genetic complexity is an important next step to understanding the role of introgression in response to environmental change.

\section{The role of demographic stochasticity}

In the model here, the potential for introgressive hybridization and persistence of the species affected by environmental change increases with demographic stochasticity: While introgressive hybridization occurs in much of the parameter range in the individualbased simulations (Figs. 3 and 4), extinction occurs 
throughout most of the same range in the deterministic simulations (Fig. 5). At first this result might seem counterintuitive given the potential for rare hybrids to be lost with demographic stochasticity, which is reflected in the greater probability of extinction rather than introgression at lower population sizes given lower carrying capacities (Figs. 3f and 4f, analogous to results from models of response to environmental change without hybridization; Boulding and Hay 2001; Orr and Unckless 2008; Gomulkiewicz and Holt 1995; Gomulkiewicz and Houle 2009). In fact, part of our original motivation for implementing an individualbased simulation was to account for the potential for demographic stochasticity to outweigh genetic effects in extinction dynamics (Lande 1988).

However, here introgressive hybridization and therefore the persistence of the species impacted by environmental change are more likely with demographic stochasticity because the initial conditions typically start that species in the basin of attraction that leads to extinction (see ESM C). In such cases, only stochasticity can randomly move the genetic composition toward the basin of attraction with introgressive hybridization and coexistence with the other species. Analogous theoretical results include the role of genetic drift in shifts between adaptive peaks (Ludwig 1981; Lande 1985) and Allee effect models where demographic stochasticity can allow population sizes to move between the extinction and persistence basins of attraction (Dennis 2002; Allen et al. 2005). Previous theory also indicates that accounting for finite individuals and populations can change the attraction to different evolutionary outcomes through an interaction of selection, drift, and the demographics of encounters (Orzack and Hines 2005). Furthermore, this result of demographic stochasticity enhancing the maintenance rather than loss of diversity (e.g., the loss of alleles through drift) echos previous individual-based models that show how demographic stochasticity can play a key role, through a variety of mechanisms, in the maintenance of diversity in general (e.g., Durrett and Levin 1994; Dieckmann and Doebeli 1999; reviewed by DeAngelis and Mooij 2005). The mechanism in Dieckmann and Doebeli (1999), where genetic drift promotes weaker association with a linked locus that influences assortative mating, has the potential to apply to the model here. In addition, this result parallels the findings of Boulding and Hay (2001) that populations could respond to a greater amount of environmental change in a model (without hybridization) that accounted for demographic stochasticity as compared to the deterministic model by Gomulkiewicz and Holt (1995), which implicitly included such dynamics with a minimum population threshold.
Similar to increasing demographic stochasticity with decreasing carrying capacity (Figs. $3 f$ and 4f), the additional density-dependent mortality with increased competition between species can quickly lead to extinction rather than successful introgression for the species affected by environmental change (Figs. 3e and 4e, especially given greater stochasticity in mating likelihood with the promiscuous mating function). This result, where greater interspecific competition might represent greater resource overlap, reflects the same general mechanism in niche evolution models (e.g., Christiansen and Loeschcke 1980; Roughgarden 1972) that requires differentiation in resource use for coexistence. Note that here we assume equal carrying capacities for both species; if we were to relax this assumption, we would expect a lower relative carrying capacity for the species affected by environmental change to reduce (or higher carrying capacity to increase) the likelihood of successful introgression through both reduced probability of finding a mate and increased interspecific competition (if present). Also, here interspecific competition depends on the species identifier, but in some cases, the allele that determines fitness in the changing environment might determine the degree of interspecific competition. For example, in the Darwin's finch study mentioned above, the introgressing trait was beak shape, which can influence competition for seeds (Grant and Grant 2002). In this case, introgression might be less likely, as individuals in the species initially affected by the environmental change that pick up the advantageous allele will then experience greater density-dependent mortality due to competition with the unaffected species. Accordingly, sympatric speciation in Dieckmann and Doebeli (1999)'s model is less likely when different (as opposed to the same) markers influence assortative mating and density-dependent interactions, where the emergence of coexisting species depends on the interaction between recombination and the genetic drift inherent in demographic stochasticity.

The other hybridization: gene flow between allopatric populations

Our results show that introgressive hybridization has the potential to rescue a species threatened with extinction after an abrupt environmental change. This process combines elements of evolutionary rescue in isolated populations that risk extinction due to a sudden environmental shift (Gomulkiewicz and Holt 1995; Boulding and Hay 2001; Orr and Unckless 2008; Tufto 2001) and of niche evolution in an open, "blackhole sink" population (Holt and Gomulkiewicz 1997; Gomulkiewicz et al. 1999; Proulx 1999; Kawecki 2000; 
Tufto 2000; Holt et al. 2003). The species initially affected by environmental change is isolated in the sense that there is no immigration from a source population or other external input of new individuals. In fact, given the resulting hybrid (Aa) offspring, hybridization depletes the abundance of homozygotes of the affected species (aa individuals) beyond the lower survival caused directly by the environmental change. Analyses of evolutionary rescue in an isolated population have stressed the importance of initial population size, rate of initial population decline, and the genetic capacity for adaptive evolution including the capacity to generate mutations favored in the new environment if the population is initially genetically depauperate (Orr and Unckless 2008). In our introgression model, the initial decline of the species affected by environmental change (aa individuals) depends on the likelihood of hybridization (Figs. $3 \mathrm{c}$ and $4 \mathrm{c}$ ) and change in fitness of the affected genotype (bb; Figs. 3a and 4a). Even though in our scenario this species is genetically uniform (aabb) when the environment first changes, favorable (B) alleles can enter the species via introgression more readily than would be possible via spontaneous mutation.

This recurrent influx of favorable alleles through introgressive hybridization in sympatric populations is analogous to immigration from allopatric populations experiencing differential selection, another dynamic that has recently received attention for its potential to enhance adaptive genetic diversity under some conditions (e.g., Gomulkiewicz et al. 1999; Gandon and Michalakis 2002; Alleaume-Benharira et al. 2006). Of particular relevance are black-hole sink models where migration from the source is necessary for persistence in a maladapted sink population. Analyses of such models indicate that the rate of adaptation is the greatest at intermediate levels of influx; with low immigration, favorable alleles are introduced only rarely and usually lost while high immigration can suppress their fitness with density dependence (Gomulkiewicz et al. 1999; Alleaume-Benharira et al. 2006). Our results related to the impact of interspecific mating probabilities (Figs. 3c and $4 \mathrm{c}$ ) echo this finding to the degree that the interspecific mating rate governs the rate of introgression of favorable alleles into the species affected by environmental change. Like the black-hole sink model, introgression at low probabilities of interspecific mating is too slow to allow this species to adapt. However, the reduction in successful introgression at high rates of hybridization is caused by hybrid inferiority in our model as well as density-dependent interactions between hybrids and the species unaffected by environmental change. These considerations show that, while introgressive hybridization shares similarities with evolutionary rescue in isolated populations and niche evolution in black-hole sinks, the introgressive process is clearly distinct from both and may more readily allow for successful adaptation by a species threatened by a sudden change of environment.

The literature on migration between allopatric populations also reinforces the importance of accounting for demographics in general (Holt and Gomulkiewicz 1997) and demographic stochasticity in particular (Alleaume-Benharira et al. 2006) when investigating the effect of genetic exchange between populations on self-persistence. With respect to demographics, accounting for changes in the relative proportion of immigrants with local fitness-driven changes population size is critical to understanding the role of migration (Holt and Gomulkiewicz 1997) similar to the importance of the effect of population size on the frequency of hybridization here. When accounting for demographic stochasticity, Holt et al. (2003) observed "punctuated" transients with persistence of maladapted populations at low levels for long periods before rapidly adapting and expanding, similar to the transient dynamics observed here (Fig. 2b, c). Furthermore, the increase in the potential for adaptation with demographic stochasticity as compared to the deterministic case found here (described in the previous section) is evident in models with migration as well (Alleaume-Benharira et al. 2006; Holt et al. 2004a). Finally, the reduced potential for adaptation with increased density dependence (Gomulkiewicz et al. 1999) directly parallels the results here (Figs. 3e, f and 4e, f). In Gomulkiewicz et al. (1999), this trend arises from density dependence lowering absolute fitness, suggesting a potential mechanism for this trend beyond the general effect of demographic stochasticity on the likelihood of extinction.

Given these similarities, additional results from this literature can provide insight into how more biological realistic dynamics might affect our results. For example, Proulx (1999) found that sexual selection based on fitness (individual health) increased the potential for adaptation to novel environments or black-hole sinks. In the model presented here, such sexual selection would likely also increase backcrossing of rare hybrids but might decrease initial hybridization after the environmental shift. In addition, the model by Holt et al. (2004b) indicates a potential dual role of incorporating Allee effects: While positive density dependence would likely increase the potential for extinction rather than adaptation in general (due to the additional negative effects of the population size decreasing below a critical threshold), it might augment the role of genetic 
exchange in promoting adaptation (due to the increased importance of any demographic enhancement).

\section{Conclusions and management implications}

In summary, introgressive hybridization is a diversitygenerating mechanism that, theoretically, is more likely to allow adaptive response to environmental change for species under certain conditions: (1) the environmental change affects potential to persist but not overly so, (2) the mating system has low stochasticity in mating success and allows similar individuals to find each other as well as greater hybridization with more limited mate choice, (3) assortative mating is intermediate to allow both the maintenance of separate species but also occasional hybridization, and (4) the traits affecting assortative mating and fitness in the changing environment are not strongly linked.

From a management perspective, whether or not introgressive hybridization in response to environmental change is desirable depends on the circumstances. Desirable cases will occur when the resulting adaptation has the potential to rescue a native species from extinction, such as adaptation to the sudden climate shifts that might become more frequent or extreme with climate change (Kharin and Zwiers 2005). In such cases, management actions to protect hybridization, such as the protection of hybrid zones, might enhance the potential for species to respond to environmental change. In addition to our results with environmental change (Figs. 3 and 4) indicating when such actions are more likely to be successful, our results without environmental change (Figs. B.1 and B.2 in ESM B) provide a cautionary indication of when management to actively promote introgression might inadvertently lead to the breakdown of barriers between species (e.g., if the relative hybrid fitness is too high or degree of assortative mating too low).

Hybridization and introgression can also cause conservation problems, such as hybridization and introgression between invasive and native species (Rhymer and Simberloff 1996). Such introgression can occur in either direction: For a small, newly introduced population, introduction represents a sudden environmental shift, and introgression of genes from the native to the introduced population could promote the genetic adaptation that allows the latter to become invasive (the subject of the model by Hall et al. 2006). On the other hand, introgression can occur between an established, prevalent invasive and rare native, which blurs the line of what constitutes a native species (the subject of the models by Tanaka 2007; Epifanio and Philipp 2001; Huxel 1999; Ferdy and Austerlitz 2002, which echo the importance of recombination, assortative mating, and the demographics of mate encounters found here). In either scenario, management actions to reduce hybridization, such as the sterilization of farmed or aquacultured organisms that can become invasive through escapement (Naylor et al. 2005), can reduce such impacts. For both desirable and undesirable introgression, the results presented here represent a first step toward identifying which types of species might make sense for prioritizing such management efforts.

Acknowledgements This manuscript greatly benefited from conversations with and feedback from B. R. Grant, R. D. Holt, S. Schreiber, and an anonymous reviewer. Support for this research came from the National Science Foundation Award \#DEB0918984 to M.L.B. and Awards \#DMS-0540524 and \#DEB0919376 to R.G. Finally, we thank S. A. Levin (who served as M.L.B.'s Ph.D. advisor and is R.G.'s academic grandfather), the toast of this special issue, for his constant and consistently superb mentorship.

Open Access This article is distributed under the terms of the Creative Commons Attribution Noncommercial License which permits any noncommercial use, distribution, and reproduction in any medium, provided the original author(s) and source are credited.

\section{References}

Alleaume-Benharira M, Pen IR, Ronce O (2006) Geographical patterns of adaptation within a species' range: interactions between drift and gene flow. J Evol Biol 19(1):203-215

Allen LJS, Fagan JF, Hognas G, Fagerholm H (2005) Population extinction in discrete-time stochastic population models with an Allee effect. J Differ Equ Appl 11(4-5):273-293

Anderson E (1948) Hybridization of the habitat. Evolution 2(1):1-9

Arnold M, Martin N (2010) Hybrid fitness across time and habitats. Trends Ecol Evol 25(9):530-536

Arnold ML, Sapir Y, Martin NH (2008) Genetic exchange and the origin of adaptations: prokaryotes to primates. Philos Trans R Soc Lond Ser B Biol Sci 363(1505):2813-2820

Barton NH (2001) The role of hybridization in evolution. Mol Ecol 10(3):551-568

Bearhop S, Fiedler W, Furness RW, Votier SC, Waldron S, Newton J, Bowen GJ, Berthold P, Farnsworth K (2005) Assortative mating as a mechanism for rapid evolution of a migratory divide. Science 310(5747):502-504

Boulding EG, Hay T (2001) Genetic and demographic parameters determining population persistence after a discrete change in the environment. Heredity 86:313-324

Buerkle CA, Morris RJ, Asmussen MA, Rieseberg LH (2000) The likelihood of homoploid hybrid speciation. Heredity 84(4):441-451

Chan K, Levin S (2005) Leaky prezygotic isolation and porous genomes: rapid introgression of maternally inherited DNA. Evolution 59(4):720-729

Christiansen F, Loeschcke V (1980) Evolution and intraspecific exploitative competition I. One-locus theory for small additive gene effects. Theor Popul Biol 18(3):297-313 
Courchamp F, Clutton-Brock T, Grenfell B (1999) Inverse density dependence and the Allee effect. Trends Ecol Evol 14(10):405-410

Coyne JA, Orr HA (2004) Speciation. Sinauer Associates, Sunderland

DeAngelis DL, Mooij WM (2005) Individual-based modeling of ecological and evolutionary processes. Annu Rev Ecol Evol Syst 36:147-168

Dennis B (2002) Allee effects in stochastic populations. Oikos 96(3):389-401

Dieckmann U, Doebeli M (1999) On the origin of species by sympatric speciation. Nature 400(6742):354-357

Dowling TE, Secor CL (1997) The role of hybridization and introgression in the diversification of animals. Annu Rev Ecol Syst 28:593-619

Duenez-Guzman EA, Mavarez J, Vose MD, Gavrilets S (2009) Case studies and mathematical models of ecological speciation. 4. Hybrid speciation in butterflies in a jungle. Evolution 63(10):2611-2626

Durrett R, Levin S (1994) The importance of being discrete (and spatial). Theor Popul Biol 46(3):363-394

Epifanio J, Philipp D (2001) Simulating the extinction of parental lineages from introgressive hybridization: the effects of fitness, initial proportions of parental taxa, and mate choice. Rev Fish Biol Fish 10(3):339-354

Felsenstein J (1981) Skepticism towards Santa Rosalia, or why are there so few kinds of animals. Evolution 35(1):124-138

Ferdy J, Austerlitz F (2002) Extinction and introgression in a community of partially cross-fertile plant species. Am Nat 160(1):74-86

Frankham R, Kingsolver J (2004) Responses to environmental change: adaptation or extinction. In: Ferrière R, Dieckmann U, Couvet D (eds) Evolutionary conservation biology. Cambridge University Press, Cambridge, pp 85100

Gandon S, Michalakis Y (2002) Local adaptation, evolutionary potential and host-parasite coevolution: interactions between migration, mutation, population size and generation time. J Evol Biol 15(3):451-462

Gavrilets S (2004) Fitness landscapes and the origin of species, monographs in population biology, vol 41. Princeton University Press, Princeton

Gavrilets S, Boake C (1998) On the evolution of premating isolation after a founder event. Am Nat 152(5):706-716

Gomulkiewicz R, Holt RD (1995) When does evolution by natural-selection prevent extinction. Evolution 49(1):201207

Gomulkiewicz R, Houle D (2009) Demographic and genetic constraints on evolution. Am Nat 174(6):E218-E229

Gomulkiewicz R, Holt RD, Barfield M (1999) The effects of density dependence and immigration on local adaptation and niche evolution in a black-hole sink environment. Theor Popul Biol 55(3):283-296

Gomulkiewicz R, Holt R, Barfield M, Nuismer S (2010) Genetics, adaptation, and invasion in harsh environments. Evol Appl 3(2):97-108

Grant BR, Grant PR (2008) Fission and fusion of Darwin's finches populations. Philos Trans R Soc Lond Ser B Biol Sci 363(1505):2821-2829

Grant PR, Grant BR (2002) Unpredictable evolution in a 30-year study of Darwin's finches. Science 296(5568):707-711

Hall RJ, Hastings A, Ayres DR (2006) Explaining the explosion: modelling hybrid invasions. Proc R Soc Lond Ser B Biol Sci 273:1385-1389
Holt RD, Gomulkiewicz R (1997) How does immigration influence local adaptation? A reexamination of a familiar paradigm. Am Nat 149(3):563-572

Holt R, Gomulkiewicz R, Barfield M (2003) The phenomenology of niche evolution via quantitative traits in a 'blackhole' sink. Proc R Soc Lond Ser B Biol Sci 270(1511): 215-224

Holt RD, Barfield M, Gomulkiewicz R (2004a) Temporal variation can facilitate niche evolution in harsh sink environments. Am Nat 164(2):187-200

Holt RD, Knight TM, Barfield M (2004b) Allee effects, immigration, and the evolution of species' niches. Am Nat 163(2):253-262

Huxel GR (1999) Rapid displacement of native species by invasive species: effects of hybridization. Biol Conserv 89(2):143-152

Karlin S, McGregor J (1972) Polymorphisms for genetic and ecological systems with weak coupling. Theor Popul Biol 3(2):210-238

Karlin S, Scudo FM (1969) Assortative mating based on phenotype: II. Two autosomal alleles without dominance. Genetics 63(2):499-510

Kawecki T (2000) Adaptation to marginal habitats: contrasting influence of the dispersal rate on the fate of alleles with small and large effects. Proc R Soc Lond Ser B Biol Sci 267(1450):1315-1320

Kharin VV, Zwiers FW (2005) Estimating extremes in transient climate change simulations. J Clim 18(8):1156-1173

Lande R (1985) Expected time for random genetic drift of a population between stable phenotypic states. Proc Natl Acad Sci U S A 82(22):7641-7645

Lande R (1988) Genetics and demography in biological conservation. Science 241(4872):1455-1460

Levin SA (1992) The problem of pattern and scale in ecology. Ecology 73(6):1943-1967

Lewontin RC, Birch LC (1966) Hybridization as a source of variation for adaptation to new environments. Evolution 20(3):315-336

Lewontin RC, Kojima K (1960) The evolutionary dynamics of complex polymorphisms. Evolution 14(4):458-472

Ludwig D (1981) Escape from domains of attraction for systems perturbed by noise. In: Enns RH, Jones BL, Miura RM, Rangnekar SS (eds) NATO ASIB proc. 75: Nonlinear phenomena in physics and biology, pp 549-566

Lynch M, Lande R (1993) Evolution and extinction in response to environmental change. In: Kareiva PM, Kingsolver JG, Huey RB (eds) Biotic interactions and global change, Sinauer Associates, Sunderland, pp 234-250

Lynch M, Gabriel W, Wood AM (1991) Adaptive and demographic responses of plankton populations to environmental-change. Limnol Oceanogr 36(7):1301-1312

McCarthy EM, Asmussen MA, Anderson WW (1995) A theoretical assessment of recombinational speciation. Heredity 74:502-509

Naylor R, Hindar K, Fleming IA, Goldburg R, Williams S, Volpe J, Whoriskey F, Eagle J, Kelso D, Mangel M (2005) Fugitive salmon: assessing the risks of escaped fish from net-pen aquaculture. BioScience 55(5):427-437

O'Donald $\mathrm{P}$ (1960) Assortive mating in a population in which two alleles are segregating. Heredity 15:389-396

Orr HA, Unckless RL (2008) Population extinction and the genetics of adaptation. Am Nat 172(2):160-169

Orzack SH, Hines WGS (2005) The evolution of strategy variation: will an ESS evolve? Evolution 59(6):1183-1193 
Pease CM, Lande R, Bull JJ (1989) A model of population growth, dispersal, and evolution in a changing environment. Ecology 70(6):1657-1664

Proulx S (1999) Matings systems and the evolution of niche breadth. Am Nat 154:89-98

Reznick DN, Ghalambor CK (2001) The population ecology of contemporary adaptations: what empirical studies reveal about the conditions that promote adaptive evolution. Genetica 112113:183-198

Rhymer J, Simberloff D (1996) Extinction by hybridization and introgression. Annu Rev Ecol Syst 27(1):83-109

Rieseberg LH, Raymond O, Rosenthal DM, Lai Z, Livingstone K, Nakazato T, Durphy JL, Schwarzbach AE, Donovan LA, Lexer C (2003) Major ecological transitions in wild sunflowers facilitated by hybridization. Science 301(5637):1211-1216

Roughgarden J (1972) Evolution of niche width. Am Nat 106(952):683-718
Stephens PA, Sutherland WJ, Freckleton RP (1999) What is the Allee effect? Oikos 87(1):185-190

Stockwell CA, Hendry AP, Kinnison MT (2003) Contemporary evolution meets conservation biology. Trends Ecol Evol 18:94-101

Svirezhev YM (1968) The systems of weakly connected populations. Stud Biophys 10:25-30

Tanaka Y (2007) Introgressive hybridization as the breakdown of postzygotic isolation: a theoretical perspective. Ecol Res 22:929-939

Tufto J (2000) Quantitative genetic models of the balance between migration and stabilizing selection. Genet Res 76:283293

Tufto J (2001) Effects of releasing maladapted individuals: a demographic-evolutionary model. Am Nat 158(4):331-340

Wirtz P (1999) Mother species-father species: unidirectional hybridization in animals with female choice. Anim Behav 58(1):1-12 